\title{
BMJ open A multicentre RCT on community occupational therapy in Alzheimer's disease: 10 sessions are not better than one consultation
}

\author{
Sebastian Voigt-Radloff, ${ }^{1}$ Maud Graff, ${ }^{2}$ Rainer Leonhart, ${ }^{3}$ Katrin Schornstein, ${ }^{3}$ \\ Frank Jessen, ${ }^{4}$ Jens Bohlken, ${ }^{5}$ Brigitte Metz, ${ }^{6}$ Andreas Fellgiebel, ${ }^{7}$ Richard Dodel, ${ }^{8}$ \\ Gerhard Eschweiler, ${ }^{9}$ Myrra Vernooij-Dassen, ${ }^{10}$ Marcel Olde Rikkert, ${ }^{11}$ \\ Michael Hüll ${ }^{12}$
}

To cite: Voigt-Radloff $S$, Graff M, Leonhart R, et al. A multicentre RCT on community occupational therapy in Alzheimer's disease: 10 sessions are not better than one consultation. BMJ Open 2011;1:e000096. doi:10.1136/bmjopen-2011000096

- Prepublication history and additional material for this paper are available online. To view these files please visit the journal online (http:// bmjopen.bmj.com).

Received 8 February 2011 Accepted 16 June 2011

This final article is available for use under the terms of the Creative Commons Attribution Non-Commercial 2.0 Licence; see http://bmjopen.bmj.com

For numbered affiliations see end of article.

Correspondence to Dr Sebastian Voigt-Radloff; sebastian.voigt@ uniklinik-freiburg.de

\section{ABSTRACT}

Objective: To compare the benefits and harms of a Dutch 10-session Community Occupational Therapy programme for patients with Alzheimer's disease with the impact of a one session consultation at home in German routine healthcare.

Design: A seven-centre, parallel group, active controlled randomised controlled trial. Patients and carers were not masked. Assessors were fully blind for treatment allocation for one of two primary-outcome measurements.

Setting: Patients' homes.

Participants: Patients with mild to moderate Alzheimer's disease (Mini-Mental State Examination 14-24), living in the community with primary carer available and without severe depression or behavioural symptoms, were eligible.

Interventions: Experimental 10 home visits within 5 weeks by an occupational therapist, educating patients in the performance of simplified daily activities and in the use of aids to compensate for cognitive decline; and educating carers in coping with behaviour of the patient and in giving supervision to the patient. Control one home visit including individual counselling of patient and carer and explanation of a leaflet on coping with dementia in daily life.

Outcome measures: The primary outcome was the patient's daily functioning measured with the Interview of Deterioration in Daily activities in Dementia and the Perceive, Recall, Plan and Perform System of Task Analysis. Assessments were at baseline, 6, 16 and 26 weeks, with a postal assessment at 52 weeks.

Results: 141 patients were 1:1 randomised to the experimental $(\mathrm{N}=71)$ and control group $(\mathrm{N}=70)$. Data for 54 and 50 participants were analysed. Patients' daily functioning did not differ significantly between the experimental and control group at week 6, 16, 26 or 52 and remained stable over 26 weeks in both groups. No adverse events were associated with the interventions.

Conclusions: In German healthcare, a Dutch 10session community occupational therapy was not better than a one-session consultation for the daily

\section{ARTICLE SUMMARY}

Article focus

- Efficiency of community occupational therapy in dementia.

- Pragmatic multicentre RCT in routine care context.

Key messages

- A 10-session community occupational therapy programme did not work more effectively than a comprehensive one-session occupational therapy consultation within German routine healthcare.

Strengths and limitations of this study

- The main strength of this trial was an elaborate multicentre RCT design within a routine care setting using an active control group, a prolonged follow-up and a strategy of video rating with fully blind assessors. However, the patients and carer could not be masked.

- The main limitation was that the training time for the interventionists was shorter, and that they had less treatment experience with the experimental intervention than the therapists of the Dutch original trial.

- Furthermore, we had to exclude 37 patients $(26 \%)$ from the multivariate analysis of variance of the primary outcome because they withdrew; or the assessment at one or more time points was missing or not within the planned time period. However, the attrition in both groups did not demonstrate a systematic bias; the analysis of the reduced patient sample with valid data did not show a tendency to significant group differences; and an additional mixed model analysis of all randomised patients did not reveal any significant differences. Consequently, the hypothesis of better effects within the experimental group must be rejected. 
functioning of patients with Alzheimer's disease. Further research on the transfer of complex psychosocial is needed.

International Clinical Trials Registry Platform:

DRKS00000053; Funded by the German Federal Ministry of Health.

\section{INTRODUCTION}

Alzheimer's disease causes high healthcare costs and burdens patients and carers with severe problems in Activities of Daily Living (ADL). ${ }^{2}$ Consequently, the improvement or the preservation of ADL is evaluated as a patient-related outcome in clinical trials related to dementia. ${ }^{3}$ ADL, burden of care, ability to stay in the community and quality-of-life issues are probably much more relevant to patients and carers than the deceleration of cognitive decline, another patient-related outcome. ${ }^{4}$ A synopsis of four systematic reviews analysing 73 RCTs on the efficacy of pharmacological and psychosocial interventions regarding everyday functioning in dementia concluded that positive effects of drugs on ADL are small (pooled effect sizes <0.28) and heterogeneous regarding safety. In contrast to the welldocumented results for pharmacological interventions, evidence for psychosocial interventions on $\mathrm{ADL}$ is lacking. ${ }^{5}$ However, a recent Dutch mono-centre RCT demonstrated significant positive effects of occupational therapy on ADL (effect sizes of 2.4, p<0.0001). ${ }^{6}$ Therefore, the purpose of our multicentre RCT was to transfer the Dutch community occupational therapy programme in a broader context of German routine healthcare and to evaluate its effectiveness and safety in comparison with an active-control-group intervention.

Occupational therapy specialises in supporting independence in ADL and is recommended in several guidelines for dementia management. ${ }^{7-9}$ Occupational therapy uses a combined approach including activity simplification, environmental modification, adaptive aids, problem-solving strategies, skill training and carer training. ${ }^{7} 1011$ According to the bio-psycho-social health model of the WHO, the negative impact of cognitive deficits on activities can be diminished by improving the patient's physical and social environment and by tailoring the intervention to the patient's capability. ${ }^{12-15}$

Until July 2011, there was no systematic review on community occupational therapy for people with Alzheimer's disease, but two research groups had conducted RCTs in this subject. In the USA study, occupational therapy demonstrated beneficial effects on patients' challenging behaviours but not on ADL. No information on adverse events was given. ${ }^{14} 16-18$ In the Netherlands, occupational therapy, tailored to the needs of patients and carers, showed benefits in the patient's ADL, mood, health status and quality of life and in the carer's sense of competence, mood, quality of life and costs of informal care. No adverse events were reported in either intervention or control group. ${ }^{6} 1920$

In the current randomised trial, we tested the hypothesis that the Dutch 10-session Community Occu- pational Therapy in Dementia Programme (COTiD) would significantly improve the daily functioning of people with mild or moderate dementia, more so than a one-session Community Occupational Therapy Consultation (COTC). Secondary research questions were whether these interventions would show any difference in their effect on patient's and primary carer's quality of life and mood; on the carer's sense of competence in the interaction with the patient; and on long-term nursing-home placements.

\section{METHODS}

Design

In order to evaluate the superiority of COTiD, we used a seven-centre single-blind, active-controlled design with a 1:1 randomisation for two parallel groups. There was no modification in design or eligibility criteria from the study protocol available at http://www.ncbi.nlm. nih.gov/pmc/articles/PMC2761388/. The study was registered at the German register of clinical trials, which is connected to the International Clinical Trials Registry Platform (http://apps.who.int/trialsearch/ $=>$ DRKS00000053).

\section{Participants and setting}

Patients were eligible to participate in the study if they had mild to moderate dementia (Mini-Mental State Examination (MMSE) 14-24) and were diagnosed as having Alzheimer's disease or mixed-type dementia, according to Tenth Revision of the International Classification of Diseases criteria, by physicians with more than 5 years of experience in dementia diagnosis. Participants had to live in the community either together with their primary carer or with involvement of a carer providing care at least twice a week. Patients with a score above 12 on the 30-items Geriatric Depression Scale or a major need of physical nursing care of more than 120 min per day (level 2 or higher according to the German LongTerm Care Insurance Act) were excluded. Unstable medical conditions or severe behavioural disturbances, which did not allow participation in the study as judged by the study physicians, were criteria for exclusion as well as for discontinuation. Long-term nursing-home placements of the patients during the treatment phase or death of patient or primary carer were criteria for discontinuation. The patient gave written informed consent, and the carer consented by written format to join and support the treatment procedures.

Patients were recruited from five outpatient memory centres at university hospitals (in Bonn, Freiburg, Mainz, Marburg and Tübingen); one municipal hospital in Karlsruhe specialising in geriatric medicine; and one neurological private practice in Berlin specialising in neuropsychiatry and collaborating with an occupational therapy private practice. The seven participating centres are located throughout Germany in urban regions with catchment areas of about 70000 to 700000 inhabitants. They had all provided outpatient dementia care for $3-15$ years. Their standard service comprised diagnostic 
work-up for dementia and related diagnoses as well as recommendation of risk reduction, dementia medication and non-pharmacological treatments. Principal investigators of the centres were psychiatrists, neurologists or geriatricians with 6-13 years of experience in dementia care.

\section{Interventions}

The experimental intervention (COTiD) was designed to improve the patient's and the primary carer's daily functioning, and was based on an evidence-based treatment manual. ${ }^{6}{ }^{19-23} \mathrm{COTiD}$ consisted of 10 occupational therapy sessions of 1 hour's duration held over 5 weeks at each patient's home. In the diagnostic phase, comprising three or four sessions, the occupational therapist explored (1) the patient's preferences and history of daily activities; (2) their ability to perform activities and to use compensatory strategies within the familiar environment; (3) the possibilities of modifying the patient's home; (4) the carer's activity preferences, problems in care giving, coping strategies and abilities to supervise; and (5) the interaction between carer and patient. In a shared decision-making process during the goal setting session, the patient and the carer selected the one or two most meaningful activities out of a list of their preferences for daily activities to work on in occupational therapy. During the treatment phase of five to six sessions, the occupational therapist defined, together with the patient and the carer, more effective compensatory and environmental strategies to adapt both the environment and the selected activities to the patient's habits and cognitive abilities. Patient and carer were taught how to use these suggested adaptations within strategies, activities and the environment in order to improve their performance of daily activities. In addition, the carer received practical and emotional support and was coached in effective supervision, problem-solving and coping strategies by means of cognitive-behavioural interventions. Detailed description of the experimental intervention has been published elsewhere. ${ }^{23}$

For the German RCT, MG taught the content of the translated treatment manual to 14 study-participant occupational therapists in $16 \mathrm{~h}$ of seminars using presentation, videos and role play with feedback and group discussion. After the seminar and before the study started, they needed to complete a full treatment series for at least one pilot dyad of patient and carer. In the study phase, the interventionists spent about $20 \mathrm{~h}$ per patient for a full treatment series including 10 treatment sessions, travel, reports and a multidisciplinary briefing. In Germany, a series of $10-30$ sessions is within the normal range of time that occupational therapists use for the treatment of older outpatients diagnosed as having other diseases, such as stroke or rheumatoid arthritis.

The control group received $1 \mathrm{~h}$ of COTC at the patient's home conducted by the same study interventionists. Based on material of the German Alzheimer's Society, two occupational therapists with more than
5 years of experience in dementia care had prepared a leaflet of 10 pages. $^{24}{ }^{25}$ The semistructured consultation was an explanation of $30 \mathrm{~min}$ of this leaflet and a talk of $30 \mathrm{~min}$ on individual problems that arose from the patient's and carer's needs. This included encouragement to stay active in everyday life, to maintain social contacts and to use dementia services in the region for which local addresses were listed in the leaflet. Occupational therapists were taught the control intervention within a $4 \mathrm{~h}$ seminar. Consultations of $30 \mathrm{~min}$ up to $1 \mathrm{~h}$ duration about such issues are common in German dementia care. A detailed description of the control intervention as well as means of quality assurance in experimental and control intervention has been published elsewhere. ${ }^{26}$

\section{Outcome measures}

The primary endpoint was the patients' change in daily functioning from baseline to follow-up time points at week 6,16 and 26 measured with the performance scale of the Interview for Deterioration in Daily Living Activities in Dementia (IDDD). ${ }^{27}$ This scale records the carer rating of the patient's need for assistance in the performance of (1) washing oneself, (2) making tea or coffee, (3) dressing, (4) combing one's hair and brushing one's teeth, (5) eating, (6) using the toilet, (7) shopping, (8) using the telephone, (9) preparing a meal, (10) cleaning the house or doing minor repair work and (11) handling finances. Each item is rated never $=0$, seldom $=1$, sometimes $=2$, often $=3$ or always $=4$. The sum of scores ranged from 0 to 44 . Higher scores indicated a higher need for assistance. Since the carer rating could not be 'masked,' daily functioning was additionally evaluated by external raters fully 'blind' to the group assignment. They rated videotapes of a challenging daily living task and used the Perceive, Recall, Plan and Perform System of Task Analysis (PRPP).$^{28}$ For the PRPP, raters had to define single steps of the performed activity, and they identified any activity step in which errors of accuracy, omission, repetition or timing occurred. The number of activity steps rated as incorrectly performed was divided by the total number of activity steps, resulting in an independence score indicated as a percentage $(100 \%=$ all steps are error-free; table 1).

Secondary endpoints included mood, quality of life, resource utilisation and possible harms (table 1). Assessors 'blind' for the group assignment, completed measurements at the patient's home at baseline and at weeks 6,16 and 26, and arranged a postal survey of carer questionnaires at week 52. The assessors had a minimum of 1 year's professional experience with older or cognitively impaired people. They attended an introductory seminar of $8 \mathrm{~h}$. The complete assessment was applied during a $2 \mathrm{~h}$ visit at each patient's home including (1) handing out and explaining the questionnaires to the carer; (2) interviewing the patient (Dementia Quality of Life Instrument and Short-Form 12 Health Survey Questionnaire) in a separate room; (3) videotaping the patient; and (4) receiving back the carer questionnaires, 
Table 1 Measurements of secondary endpoints ${ }^{26}$

\begin{tabular}{ll}
\hline Endpoint & Measurement \\
\hline $\begin{array}{l}\text { Patient's initiative in daily activities } \\
\text { Patient's mood }\end{array}$ & Interview for Deterioration in Daily Living Activities in Dementia, initiative scale \\
Carer's mood & Cornell Scale for Depression in Dementia \\
Patient and carer's quality of life & Center for Epidemiologic Depression Scale \\
& Sementia Quality of Life Instrument, overall item \\
& Short-Form 12 Health Survey Questionnaire physical \\
Carer's interaction with patient & Short-Form 12 Health Survey Questionnaire mental \\
Care by primary carer & Sense of Competence Questionnaire \\
Nursing home placement & Resource Utilisation in Dementia, hours per day \\
Harms & Resource Utilisation in Dementia, nights in nursing home (except respite care) \\
& No of adverse events \\
\hline
\end{tabular}

checking it and clarifying answers if necessary. Seminar descriptions and means of quality management for assessment as well as detailed scheme and psychometric properties of all measurement instruments have been reported recently. ${ }^{26}$

All measurement instruments are validated and used in dementia research. ${ }^{29}{ }^{30}$ For the present study, we translated the IDDD into German according to high methodological standards with two independent forward and backward translations, analysis of discrepancies and final agreement by discussion with all translators. There was no need to translate the PRPP because it was established in The Netherlands and applied by Dutch raters. There was one protocol amendment before recruitment started. The Assessment of Motor and Process Skills was replaced by the PRPP, because the Assessment of Motor and Process Skills was not available in the German language within the planned schedule.

Indicators of harm were defined as patient or carer death, number of patients with admission to hospital and number of nights in hospital. These indicators were recorded in interviews with the carer at intervals of 5-7 weeks over 52 weeks. Study sites had to report severe adverse events to the study centre immediately when each occurred. We did not assume a direct association between the defined harms and either the experimental or the control intervention. However, increased daily activities in the interventions group might have resulted in a higher risk of falls or accidents and thus may indirectly have led to more nights in hospital or, in the worst case, to death.

\section{Sample-size calculation}

A sample size of 42 participants per group was calculated to be necessary to detect an effect size of $f=0.10$ on the IDDD performance scale in an analysis of variance of two groups and four time points, using a two-sided 5\% significance level, a power of $80 \%$ and a correlation of 0.7 between the measurement time points. ${ }^{31}$ According to the Dutch original RCT, we expected a dropout rate of $10 \%$ at week 16 , which was extrapolated to $40 \%$ at week 52. A 9-month inclusion period was anticipated as necessary in order to recruit the 140 patients. Our assumed effect size of $\mathrm{f}=0.10$ is based on a group-by-time interaction and compatible with Cohen's $d=0.20$, which corresponds to a small effect size, and any d over 0.8 is large. Although the Dutch original RCT found effect sizes of $d=2.4$ in the IDDD performance scale at week 12 , for this study the power was calculated much more conservatively.

This was because we (1) introduced an active control group, (2) investigated the programme effects under varying care conditions in seven centres with interventionists who were introduced in this new treatment and were not as experienced as the Dutch study therapists and (3) prolonged the follow-up period. Interim analyses were not planned.

\section{Randomisation and masking}

The random allocation sequence was computer-generated with blocking by centre and groups of two persons, without stratification and in a ratio of $1: 1$ by a statistician from a distant site. After enrolment, study site physicians requested randomisation via email. The statistician emailed the individual allocation to COTiD or COTC exclusively to the site interventionist and stored the allocation list at his distant site which was not available to any study site staff. The interventionist scheduled treatment sessions, faxed records to the distant coordinating study centre and kept all documents strictly separated from any other site staff. This was in order to avoid contamination. Since the numbers of home visits differed in the experimental and control groups, masking of patients and carers was not possible. However, study information did not include any preference for a special treatment 'arm.' Patients and carers were asked to give no information about their treatment package to assessors or study physicians. All study personnel were 'blind' for group assignment, except the interventionists. Agreement between the assessors' estimation of group assignment and the actual group assignment was $61 \%$, and thus slightly over the expected $50 \%$ of agreement by chance. The procedure of external video rating ensured the full 'blinding' of the external raters for the PRPP primary outcome measure. Independent research assistants cleaned the videotapes of 
any hint of group assignment before they were rated by two Dutch raters not involved in the trial treatment. In order to establish the inter-rater reliability, we tested 10 double ratings of the same video by the two raters and found an intraclass correlation coefficient of 0.9. Data analysts were not 'blind' for the group assignment. However, measurement time points and outcomes had been published before data were available for analysis ${ }^{26}$ and any decision to remove patients from the analyses is reported in the present publication.

\section{Statistical methods}

Data were entered via special MS Access entry masks automatically controlling for data plausibility. In addition, sections of entered data were checked for typing errors by hand, in order to ensure an error rate lower than $0.2 \%$. The primary intention-to-treat analysis included all allocated participants with valid data whether they did or did not receive the complete intervention. For the IDDD and the PRPP measurements of the primary outcome, we performed a multivariate analysis of variance (MANOVA) with repeated measures with two groups and four measurement time points at baseline, weeks 6, 16 and 26. A univariate ANOVA with five measurement time points (+postal assessment in week 52) was carried out for the secondary outcomes and the IDDD. We did not adjust for baseline values, because we found no marked group differences. In order to deal with missing data occurring not in the primary but in the secondary outcomes, we performed secondary intention-to-treat analyses with multiple data imputation using the Full Information Maximum Likelihood method. ${ }^{32}$ We imputed data for all secondary outcome measurements and all time points using SPSS (V.19). In an additional analysis, we used the linear mixed-effects models (MIXED) procedure in SPSS, which allows an unequal number of repetitions and a better handling of missing values.

All statistical tests were two-sided on an $\alpha$ level of 0.05 . Subgroup analyses were not planned.

\section{RESULTS}

\section{Recruitment and participant flow}

We prolonged the planned recruitment period from August 2008 to April 2009 by one additional month, up to May 2009. This was in order to recruit the intended sample size. The 52-week follow-up was closed in May 2010. One hundred and forty-one participants were recruited (Berlin: 19; Bonn: 21; Freiburg: 26; Karlsruhe: 15; Mainz: 24; Marburg: 21; Tübingen: 15). The flow chart (figure 1) shows that attrition following randomisation did not lead to any significant group differences.
Figure 1 Flow of participants through the trial.

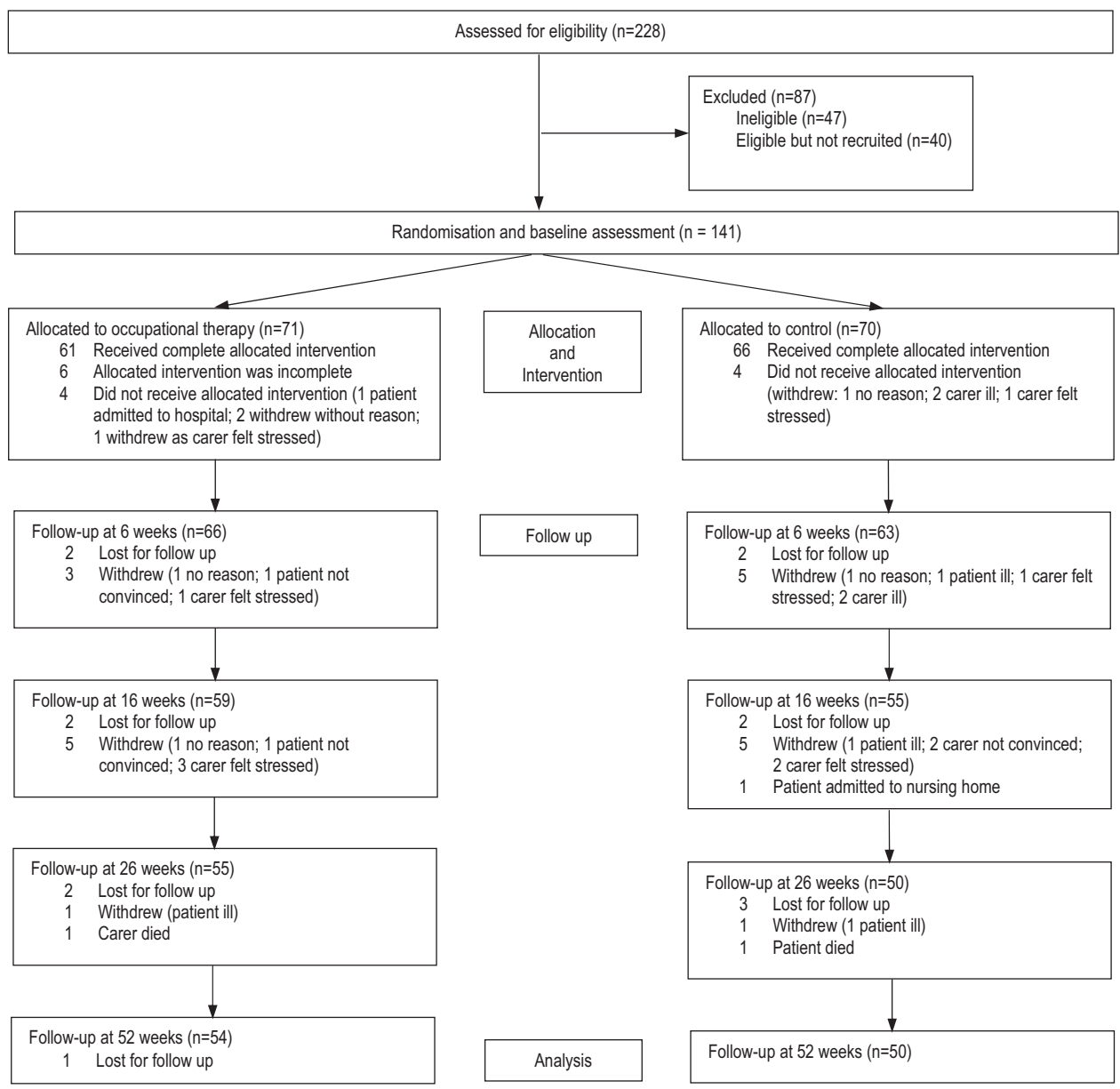




\section{Baseline characteristics}

Randomisation did avoid imbalances in baseline characteristics (table 2) and pretreatment assessment data (table 3) except in one item. Participants in the control group had more moderate to severe limitations in their financial situation ( $14 \%$ vs $2 \%$; $p=0.027$ ). Because the financial situation is not known as a predictive factor for functional decline, we did not adjust for this imbalance. $^{33}$

\section{Intervention delivery}

Sixty-one of $71(86 \%)$ allocated patient-carer dyads received complete sessions in the COTiD group, 66 of 70 (94\%) in the control group. In each group, four pairs were lost before intervention. Six patient-carer dyads in the COTiD had fewer than 10 sessions. Interventionists rated the delivery of 20 predefined treatment subprocesses, ranging from interviewing patient and carer to training of simplified activities or supporting the carer in supervision. They scored treatment delivery as $78 \%$ in the COTiD group and $80 \%$ in the control group. Interventionists rated the patient's adherence in 67 cases of the COTiD group, from 15 as hindering the delivery of treatment; 26 as neutral and 26 as facilitating. Rating criteria were the patient's cooperation during interview, goal setting and training; the daily changing mental capacity; collaboration with the carer; and the acceptance of innovations. Ratings of carers' adherence were: 5, hindering; 15, neutral; and 47, facilitating. The carer adherence was assessed with regard to the cooperation during scheduling, interview, goal setting and training to supervise; the encouragement of the patient; the acceptance of support service; and the implementation of innovations. The adherence of the participants in the control group could not be rated, because interventionists had no further contact after the consultation.

\section{Outcomes}

The MANOVA in 104 completers (COTiD: $n=54$; control: $n=50$ ) revealed no significant group-time interaction effect in the primary outcome measurements of patients' daily functioning (figures 2, 3). Using the arcsine transform ${ }^{34}$ for the PRPR percentage did not change results (original: $\mathrm{p}=0.243$; arcsine-transform: $\mathrm{p}=0.216)$. An additional mixed-models analysis of all randomised patients $(\mathrm{N}=141)$ as recommended by Coley and colleagues ${ }^{35}$ also revealed no significant interactions for the IDDD $(p=0.340)$ and the PRPP $(p=0.785)$; details are provided as supplementary online material. Tables 3, 4 show the mean, SD and group difference including 95\% CIs of an ANOVA for all outcomes. Patients' daily functioning did not change significantly over 26 weeks in either the experimental or control group. In the postal 52-week follow-up, the patients' need for assistance increased in both groups, and accordingly the carer's hours of care for basic ADL were higher. Two patients of the COTiD group were placed in nursing homes 33 and 44 weeks after baseline and one patient of the control group after 33 weeks.

To address the problem of missing data in single measurement instruments, we performed a multiple data imputation. We calculated a MANOVA over four measurement time points for all primary and secondary outcomes for all 104 completers. Ten different data

Table 2 Demographic and clinical characteristics

\begin{tabular}{|c|c|c|c|c|c|c|}
\hline & \multicolumn{3}{|c|}{$\begin{array}{l}\text { Community Occupational Therapy } \\
\text { in Dementia Programme }\end{array}$} & \multicolumn{3}{|l|}{ Control } \\
\hline & $\begin{array}{l}\text { Analysed } \\
(n=54)\end{array}$ & $\begin{array}{l}\text { Dropouts } \\
(n=17)\end{array}$ & $\begin{array}{l}\text { Total } \\
(n=71)\end{array}$ & $\begin{array}{l}\text { Analysed } \\
(n=50)\end{array}$ & $\begin{array}{l}\text { Dropouts } \\
(n=20)\end{array}$ & $\begin{array}{l}\text { Total } \\
(n=70)\end{array}$ \\
\hline Age, years (SD) & $78.0(7.1)$ & $77.2(8.5)$ & $77.8(7.4)$ & $78.7(6.0)$ & $78.3(7.1)$ & $78.5(6.3)$ \\
\hline Sex, female & $29(54 \%)$ & $12(71 \%)$ & $41(58 \%)$ & $30(60 \%)$ & $10(50 \%)$ & $40(57 \%)$ \\
\hline Mini-Mental State Examination (SD) & $20.4(3.1)$ & $19.0(3.3)$ & $20.2(3.2)$ & $20.7(2.7)$ & $20.3(2.9)$ & $20.7(2.7)$ \\
\hline Geriatric Depression Scale (SD) & $6.9(3.0)$ & $5.6(2.9)$ & $6.5(3.0)$ & $5.2(2.8)$ & $6.1(2.6)$ & $5.5(2.8)$ \\
\hline \multicolumn{7}{|l|}{ Education } \\
\hline No school graduation & $2(4 \%)$ & $1(6 \%)$ & $3(4 \%)$ & $1(2 \%)$ & $0(0 \%)$ & $1(1 \%)$ \\
\hline Middle school graduation ( 9 or 10 years) & $41(76 \%)$ & $13(76 \%)$ & $54(76 \%)$ & $37(74 \%)$ & $15(75 \%)$ & $52(74 \%)$ \\
\hline High school graduation (12 or 13 years) & $11(20 \%)$ & $3(18 \%)$ & $14(20 \%)$ & $12(24 \%)$ & $5(25 \%)$ & $17(24 \%)$ \\
\hline \multicolumn{7}{|c|}{ Financial situation as perceived by the carer } \\
\hline No limitation & $40(74 \%)$ & $14(82 \%)$ & $54(76 \%)$ & $38(76 \%)$ & $13(65 \%)$ & $51(73 \%)$ \\
\hline Minor limitation & $12(22 \%)$ & $1(6 \%)$ & $13(18 \%)$ & $3(6 \%)$ & $3(15 \%)$ & $6(9 \%)$ \\
\hline Moderate or severe limitation & $1(2 \%)$ & $2(12 \%)$ & $3(4 \%)$ & $7(14 \%)$ & $4(20 \%)$ & $11(16 \%)$ \\
\hline No data & $1(2 \%)$ & $0(0.0 \%)$ & $1(1 \%)$ & $2(4 \%)$ & $0(0 \%)$ & $2(3 \%)$ \\
\hline \multicolumn{7}{|l|}{ Primary carer } \\
\hline Age, years (SD) & $65.4(16.3)$ & $63.1(14.0)$ & $64.9(15.7)$ & $65.9(13.0)$ & $61.4(17.4)$ & $64.5(14.4)$ \\
\hline Sex, female & $38(70 \%)$ & $9(53 \%)$ & $47(66 \%)$ & $35(70 \%)$ & $18(90 \%)$ & $53(76 \%)$ \\
\hline Spouse & $32(59 \%)$ & $8(47 \%)$ & $40(56 \%)$ & $31(62 \%)$ & $9(45 \%)$ & $40(57 \%)$ \\
\hline Daughter or son (in law) & $20(37 \%)$ & $7(41 \%)$ & 27 (38\%) & $16(32 \%)$ & $9(45 \%)$ & $25(36 \%)$ \\
\hline Others & $2(4 \%)$ & $2(12 \%)$ & $4(6 \%)$ & $3(6 \%)$ & $2(10 \%)$ & $5(7 \%)$ \\
\hline Living together (\%) & $41(76 \%)$ & $11(65 \%)$ & $52(73 \%)$ & $33(66 \%)$ & $14(70 \%)$ & $47(67 \%)$ \\
\hline
\end{tabular}




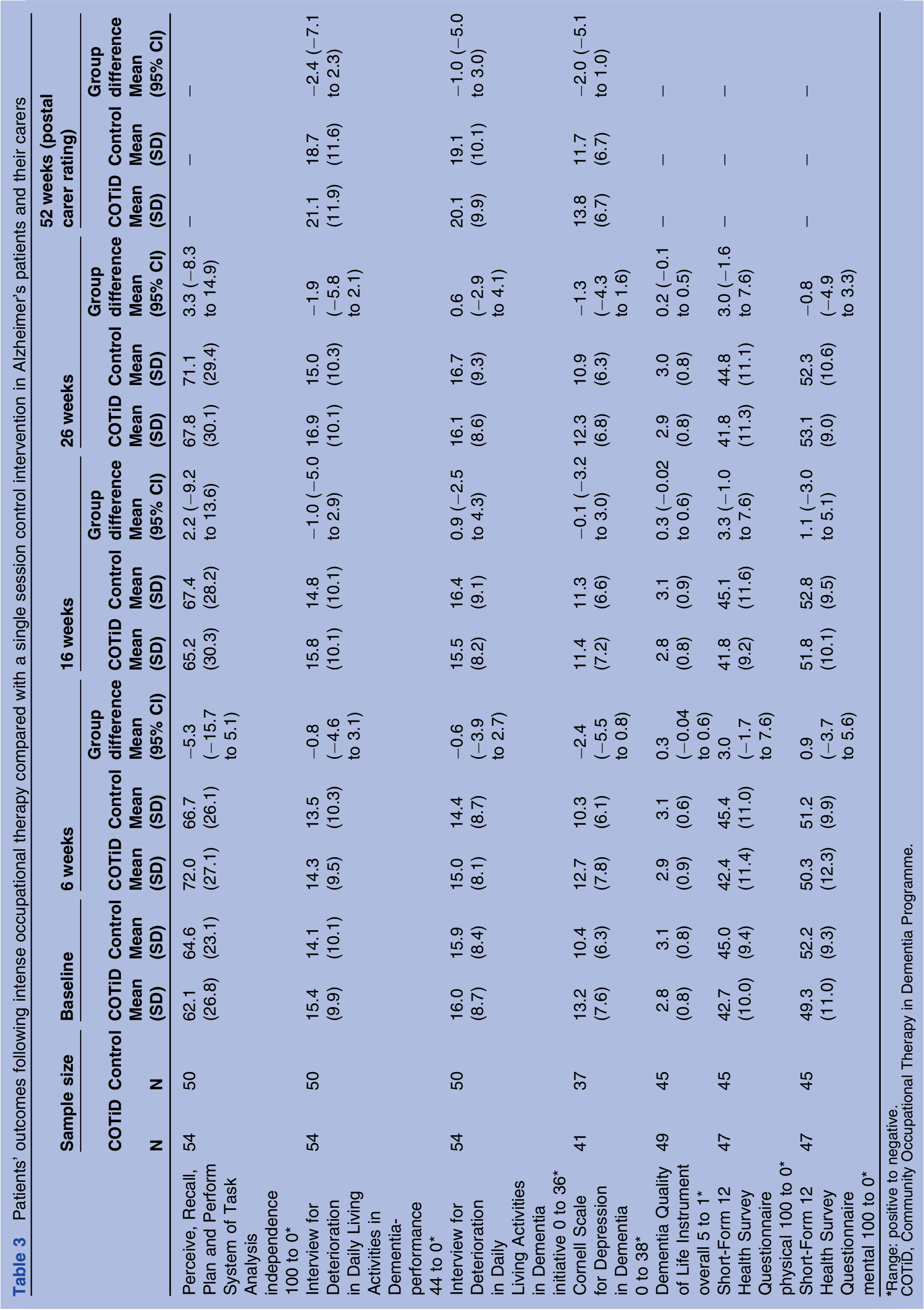




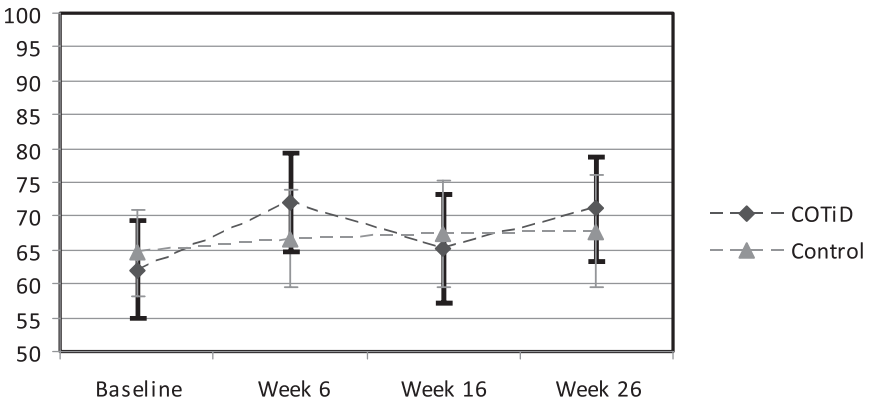

Figure 2 Activities of Daily Living task performance of Alzheimer's patients following intense occupational therapy compared with a single session control intervention; means and $95 \%$ Cls of the Perceive, Recall, Plan and Perform System of Task Analysis independence scale ( $\mathrm{N}=104$ completers; range: $100=$ no errors to $0=$ all errors). COTiD, Community Occupational Therapy in Dementia Programme.

imputations did not reveal any significant time-group interaction effects.

We also tested for study site differences at baseline and found no significant differences in a MANOVA with the factors study sites and intervention groups $(\mathrm{F}(66,432)=$ $1.079, \mathrm{p}=0.323)$. Furthermore, no study-site effect was found in the primary outcome analysing IDDD and PRPP data of baseline, and weeks 6, 16 and 26 (IDDD: $F(6,90)=0.724, p=0.631$; PRPP: $F(6,90)=$ $1.758, \mathrm{p}=0.117)$.

\section{Harms}

There were no differences between intervention and control group, either in the number of adverse events or in their severity. The study-site physicians judged all adverse events as unrelated to trial treatment or assessment contacts. In the total sample of all randomised participants $(n=141)$, two deaths of patients (both in the control group) and one death of a carer (in the COTiD group) were reported. In the COTiD group, 14 patients were admitted to hospital for an average of 15 nights, and 10 patients in the control group for an average of 18 nights. There was no difference between the two

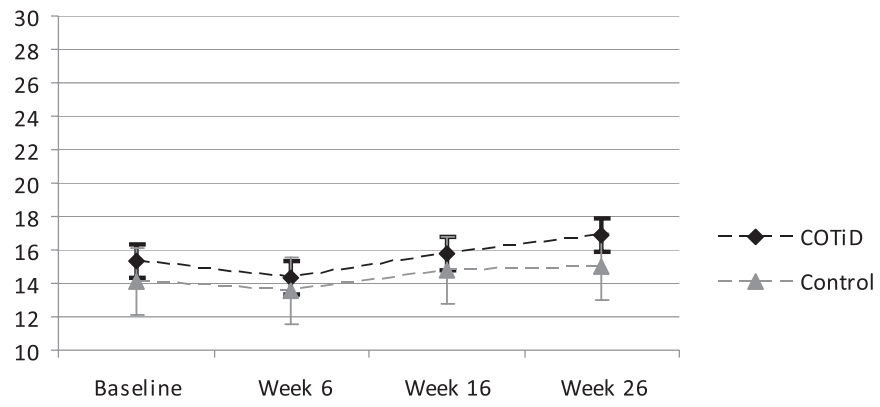

Figure 3 Need for assistance in Activities of Daily Living of Alzheimer's patients following intense occupational therapy compared with a single-session control intervention; means and $95 \%$ Cls of the Interview for Deterioration in Daily Living Activities in Dementia performance scale ( $N=104$ completers; range: $0=$ never needed assistance to $44=$ always needed assistance). COTiD, Community Occupational Therapy in Dementia Programme. groups in average number of nights admitted to hospital $(\mathrm{F}(1,97)=2.785, \mathrm{p}=0.1)$. All events were unrelated to the occupational therapy sessions.

\section{DISCUSSION}

In the results of this study, a 10-session community occupational therapy in dementia programme (COTiD) was found to be no more beneficial than a one-session consultation concerning short- and middle-term effects on patients' daily functioning. In both groups, the need for assistance in basic and instrumental ADL and the performance of a self-chosen daily living task remained stable up to 6 months after baseline. No significant group differences could be found on secondary outcomes, which were quality of life and mood of patient and primary carer; patient's initiative in daily activities; carer's sense of competence in interaction with the patient; carer's hours of daily care; and patient's nursing home placement. There were no adverse events associated with experimental or control intervention.

\section{Limitations}

Despite an elaborate study design, there are several limitations in this study. We analysed only 104 completer dyads from 141 recruited pairs (74\%). However, (1) baseline data of completers and non-completers did not show an imbalance; (2) dyads were maintained, whose data were valid, and for whom treatment was intended but not received in the complete ITT-analysis; (3) an additional mixed model analysis of all randomised patients also did not reveal any significant differences; and (4) the analysis of the reduced patient sample with valid data did not show even a tendency towards significant group differences. Thus, the hypothesis of group differences must be rejected, because the analysis of completers usually favours results in the direction of group differences.

A second shortcoming was that following the common introductory seminar, the start of the study differed among the sites due to different time lines in administrative matters and approval of the local ethic commissions. Therefore, a common repetition seminar for the interventionists could not be arranged after the pilot training. This may have led to some heterogeneity in the intervention, especially because in Germany, 11 newly introduced interventionists performed the treatment compared with two experienced experts in the original Dutch trial. We addressed this problem with feedback on videos of treatment sessions the interventionists sent in. Furthermore, we arranged telephone supervision on demand.

We consider the contamination of the control intervention with knowledge from the experimental intervention to be low, because any specific intervention such as activity selection, simplification or training was precluded by the limited time to carry out the control intervention.

\section{Comparison}

The Dutch RCT on the COTiD with waiting-controlgroup design showed large effect sizes in the IDDD performance scale at 6 and 12 weeks after baseline 


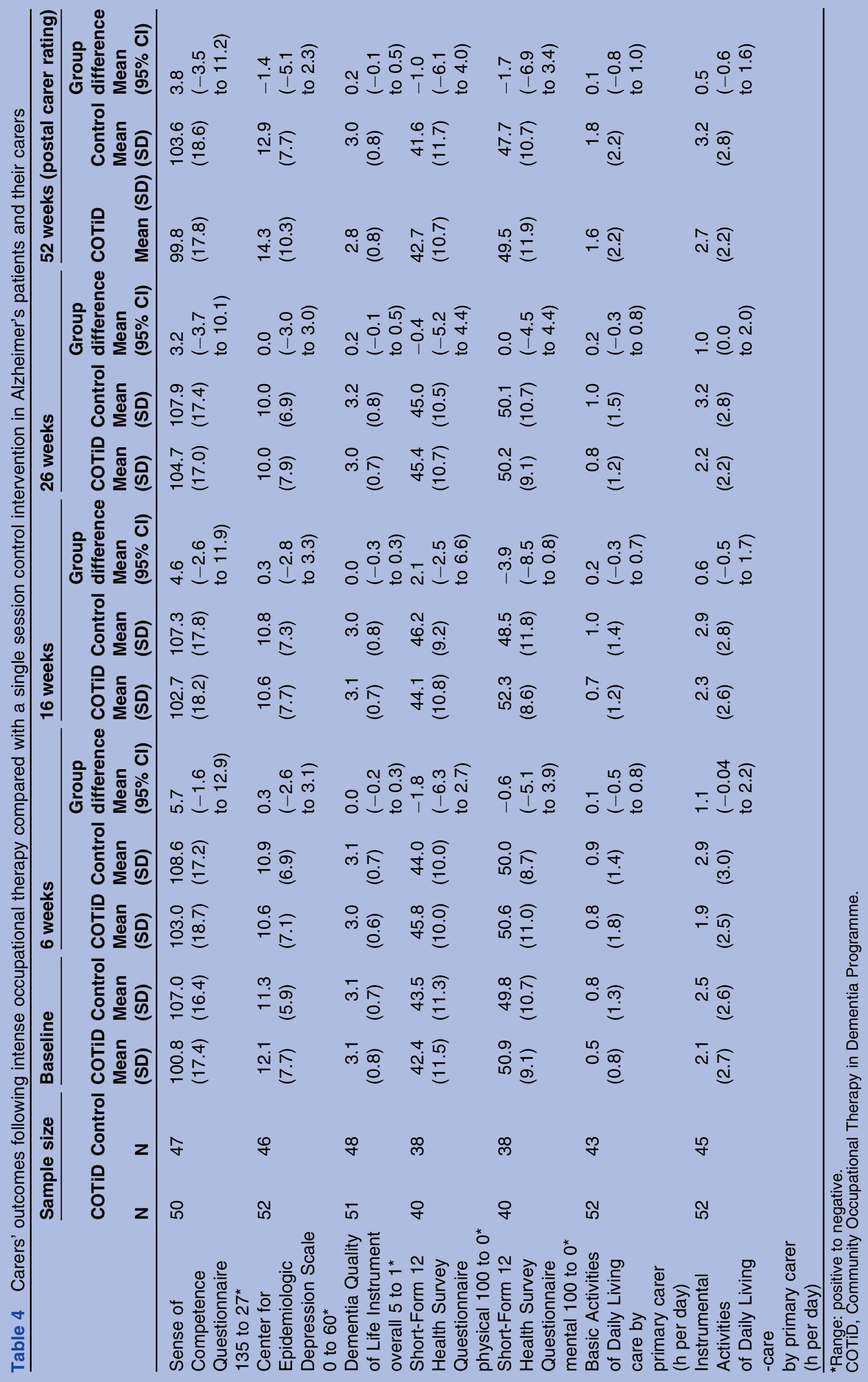


$\left(\mathrm{d}=2.3\right.$ and 2.4, respectively). ${ }^{6}$ Since the Dutch COTiD programme demonstrated such highly positive effects, we judged it as appropriate to conduct not an identical replication, but a twofold transfer from the source to the target country and from a mono-centre RCT design with high expertise of interventionists to a pragmatic multicentre RCT design in routine care ${ }^{36}$ The Dutch and the German sample did not differ remarkably in cognition at baseline (MMSE: 19 vs 20), but did differ in the need for assistance (IDDD performance: 24 vs 15). The German patients showed a low need for assistance at the beginning of the study. This was comparable with the IDDD values of the Dutch patients at the end of the treatment. This may have caused a floor effect on the IDDD. Another mono-centre RCT in the USA compared community occupational therapy and a less intensive telephone consultation in patients with probable dementia (MMSE: 13). ${ }^{37}$ The authors found a small effect size in daily functioning $(d=0.21)$. The initial need of assistance in both studies was higher than in the German sample. A systematic review of community programmes in dementia ${ }^{38}$ reported one study on exercise and behavioural management with beneficial effects on daily functioning of patients with moderate dementia (MMSE: 17); one trial on occupational therapy with heterogeneous effects; and two studies on occupational therapy and music therapy with no significant effects. A current German health-technology assessment on non-drug therapies in Alzheimer's disease did not identify any further community occupational therapy trials. ${ }^{39}$ The comparison of community intervention trials reveals that study samples with a lower MMSE and a higher need for assistance have a greater benefit than those with higher initial cognitive and daily functioning. Similarly, a standardised synopsis of ADL outcomes in pharmacological dementia trials indicated that samples with an MMSE between 17 and 10 have the greatest benefit in ADL, while samples with higher MMSE scores showed smaller effects. ${ }^{40}$ However, different baseline scores of cognitive and daily functioning alone cannot explain the major difference between the findings in this German study and the positive results of the Dutch RCT. Detailed process evaluation and exploratory analyses of the study data might show whether variations in studysite context and treatment performance influenced the intervention's effectiveness.

\section{Clinical and research implications}

Published evidence for the effectiveness of community occupational therapy in dementia is heterogeneous, as indicated by a Dutch trial with large positive effects on daily functioning, a few USA trials with no or small positive effects on ADL and this German study showing that 10 sessions were not better than one consultation. A preventive one-session consultation might be hypothesised as beneficial for people with mild dementia and an improved 10-session programme more specifically adapted to the German healthcare system as beneficial for dementia patients with a moderate need for assis- tance in ADL, as shown in the Dutch study in which most people with dementia had a moderate to high need for assistance at baseline.

Although we had expected smaller effect sizes than in the Dutch original trial due to a changed study design with (1) the introduction of an active control group, (2) a variance in treatment performance in several centres, (3) a prolonged follow-up time and (4) rigorous reduction in the analysed sample to participants with valid data, it remains surprising that no significant group difference could be found in any of the primary or secondary outcomes.

This study has shown that careful cross-national comparisons are greatly needed, especially in complex interventions, before they can be considered evidencebased and implemented effectively in other healthcare systems. Therefore, further analyses must investigate the role of interventionists' expertise and treatment performance, and the role of participants' needs and utilisation of healthcare resources, before conclusions on international implementation of this intense occupational therapy intervention can be drawn.

\section{Author affiliations}

${ }^{1}$ Department of Occupational Therapy, Centre of Geriatric Medicine and Gerontology Freiburg, University Hospital Freiburg, Freiburg, Germany

${ }^{2}$ Alzheimer Centre Nijmegen, Scientific Institute for Quality in Health Care and Department of Rehabilitation-0ccupational Therapy, Radboud University Nijmegen Medical Centre, Nijmegen, The Netherlands

${ }^{3}$ Department of Social Psychology and Methodology, University of Freiburg, Freiburg, Germany

${ }^{4}$ Department of Psychiatry and Psychotherapy, University of Bonn, Bonn, Germany

${ }^{5}$ Private Practice for Neurology, Psychiatry and Psychotherapy, Berlin, Germany

${ }^{6}$ Centre of Geriatric Medicine and Geriatric Clinic at Diakonissenkrankenhaus Karlsruhe-Rüppurr, Karlsruhe, Germany

${ }^{7}$ Department of Psychiatry and Psychotherapy, University Medical Center Mainz, Mainz, Germany

${ }^{8}$ Department of Neurology, Philipps-University Marburg, Marburg, Germany ${ }^{9}$ Department of Psychiatry and Psychotherapy, Eberhard-Karls University Tuebingen, Tübingen, Germany

${ }^{10}$ Alzheimer Centre Nijmegen, Scientific Institute for Quality in Health Care, Radboud University Nijmegen Medical Centre, Nijmegen, The Netherlands

${ }^{11}$ Department of Geriatrics, Alzheimer Centre Nijmegen, Radboud University Nijmegen Medical Centre, Nijmegen, The Netherlands

${ }^{12}$ Section of Gerontopsychiatry and Neuropsychology, Centre of Geriatric Medicine and Gerontology Freiburg, University Hospital Freiburg, Freiburg, Germany

Acknowledgements We thank all participants and interventionists for their contribution. We acknowledge C Mayers, Research Fellow, Faculty of Health \& Life Sciences, York St John University, UK, for critical reading and English correction.

Funding German Federal Ministry of Health, Reference Number: IIA5-2508FSB111/44-004.

Competing interests FJ: membership in DSMB of AC-immune; payment for lectures from Pfizer, Esai and Novartis. BM: payment for lectures from various pharmaceutical companies. RD: consultancy, grants, payment for lectures, patents and meeting expenses from various pharmaceutical companies; royalties and patents from University of Marburg. GE: grants from AC-immune and Janssen-AL. MOR: consultancy for Jansen-Cilag and Numico. MH: grants from Wyeth, Pfizer and Medivation; payment for lectures from Wyeth, Pfizer and Merz. 
Ethics approval Ethics approval was provided by the Medical Ethics Committee of the University Hospital Freiburg (no 110/08)

Contributors SVR, MG, RL, GE and MH contributed to the study conception and design. FJ, JB, BM, AF, RD, GE and MH acquired the data. SVR and KS participated in data and study management, and prepared the statistical analysis. $\mathrm{RL}$ performed the statistical analysis. SVR drafted the manuscript. $\mathrm{RL}, \mathrm{MG}, \mathrm{FJ}, \mathrm{JB}, \mathrm{BM}, \mathrm{AF}, \mathrm{RD}, \mathrm{GE}, \mathrm{MOR}, \mathrm{MVD}$ and $\mathrm{MH}$ revised the manuscript critically for important intellectual content. All authors read and approved the final manuscript.

Provenance and peer review Not commissioned; externally peer reviewed.

Data sharing statement Complete data sets can be provided on request for fellow researchers in the context of collaborative projects and publications.

\section{REFERENCES}

1. Wancata J, Musalek M, Alexandrowicz R, et al. Number of dementia sufferers in Europe between the years 2000 and 2050. Eur Psychiatry 2003; $18: 306-13$

2. Wimo A, Jonsson L, Winblad B. An estimate of the worldwide prevalence and direct costs of dementia in 2003. Dement Geriatr Cogn Disord 2006;21:175-81.

3. European Medicines Agency. Guideline on Medicinal Products for the Treatment of Alzheimer's Disease and other Dementias [Internet] London, 2008. Doc. Ref. CPMP/EWP/553/95 Rev. 1. http://www. emea.europa.eu/pdfs/human/ewp/055395en.pdf (accessed $29 \mathrm{Jul}$ 2011).

4. Georges J, Jansen S, Jackson J, et al. Alzheimer's disease in real life - the dementia carer's survey. Int J Geriatr Psychiatry 2008;23:546-51.

5. Voigt-Radloff $S$, Hüll M. Daily functioning in dementia: pharmacological and non-pharmacological interventions demonstrate small effects on heterogeneous scales: a synopsis of four health technology assessments of the German Institute for Quality and Efficiency in Health Care regarding the endpoint activities of daily living. Psychiatr Prax 2011;38:221-31 (In German).

6. Graff MJ, Vernooij-Dassen MJ, Thijssen M, et al. Community based occupational therapy for patients with dementia and their care givers: randomised controlled trial. BMJ 2006;333:1196.

7. National Collaborating Centre for Mental Health (commissioned by the Social Care Institute for Excellence and the National Institute for Health and Clinical Excellence). Dementia. A NICE-SCIE Guideline on Supporting People with Dementia and their Carers in Health and Social Care. National Clinical Practice Guideline, Number 42. London: The British Psychological Society and Gaskell, 2007.

8. German Society for Psychiatry, Psychotherapy and Neurology and German Society for Neurology. Consented Guideline 'Dementia' [Internet], 2009. http://media.dgppn.de/mediadb/media/dgppn/pdf/ leitlinien/s3-leitlinie-demenz-If.pdf (accessed 29 Jul 2011).

9. Work Group on Alzheimer's Disease and other dementias. Practice Guideline for the Treatment of Patients With Alzheimer's Disease and Other Dementias [Internet], 2007. http://www.psychiatryonline.com/ pracGuide/PracticePDFs/AlzPG101007.pdf (accessed 26 Oct 2010).

10. Steultjens EM, Dekker J, Bouter LM, et al. Evidence of the efficacy of occupational therapy in different conditions: an overview of systematic reviews. Clin Rehabil 2005;19:247-54.

11. Voigt-Radloff S. Occuaptional therapy in dementia. In: Haberstroh J, Pantel J, eds. Psychosocial Treatment in Dementia. German: AKA Verlag Heidelberg, 2011.

12. World Health Organization. International Classification of Functioning, Disability and Health (ICF) [Internet]. Geneva, 2001. http://www3.who.int/icf/icftem (accessed 29 Jul 2011).

13. Giovannetti T, Bettcher BM, Libon DJ, et al. Environmental adaptations improve everyday action performance in Alzheimer's disease: empirical support from performance-based assessment. Neuropsychology 2007:21:448-57.

14. Gitlin LN, Winter L, Burke J, et al. Tailored activities to manage neuropsychiatric behaviors in persons with dementia and reduce caregiver burden: a randomized pilot study. Am J Geriatr Psychiatry 2008;16:229-39.

15. Mittelman MS, Haley WE, Clay OJ, et al. Improving caregiver wellbeing delays nursing home placement of patients with Alzheimer disease. Neurology 2006;67:1592-9.

16. Gitlin LN, Corcoran M, Winter L, et al. A randomized, controlled trial of a home environmental intervention: effect on efficacy and upset in caregivers and on daily function of persons with dementia. Gerontologist 2001:41:4-14.

17. Gitlin LN, Hauck WW, Dennis MP, et al. Maintenance of effects of the home environmental skill-building program for family caregivers and individuals with Alzheimer's disease and related disorders. J Gerontol A Biol Sci Med Sci 2005;60:368-74

18. Gitlin LN, Winter L, Vause Earland T, et al. The Tailored Activity Program to reduce behavioral symptoms in individuals with dementia: feasibility, acceptability, and replication potential. Gerontologist 2009;49:428-39.

19. Graff MJ, Vernooij-Dassen MJ, Thijssen M, et al. Effects of community occupational therapy on quality of life, mood, and health status in dementia patients and their caregivers: randomized controlled trial. J Gerontol A Biol Sci Med Sci 2007;62:1002-9.

20. Graff MJ, Adang EM, Vernooij-Dassen MJ, et al. Community occupational therapy for older patients with dementia and their care givers: cost effectiveness study. BMJ 2008;336:134-8.

21. Graff MJ, Melick van MB. The development, testing and implementation of an occupational therapy guideline. The guideline for the OT diagnosis and treatment of older persons with cognitive impairments. Nederlands Tijdschrift voor Ergotherapie 2000;28:169-74 (in Dutch).

22. Graff MJ, Vernooii-Dassen MJ, Hoefnagels WH, et al. Occupational therapy at home for older individuals with mild to moderate cognitive impairments and their primary caregivers: a pilot study. OTJR: Occupation, Participation and Health 2003;23:155-63.

23. Graff MJ, Vernooij-Dassen MJ, Zajec J, et al. How can occupationa therapy improve the daily performance and communication of an older patient with dementia and his primary caregiver? A case study. Dementia 2006;5:503-32.

24. German Alzheimer Society. Berlin: Leben mit Demenzkranken, 2003 (in German).

25. German Alzheimer Society. Berlin: Ratgeber Häusliche Versorgung Demenzkranker, 2006 (in German).

26. Voigt-Radloff S, Graff M, Leonhart R, et al. WHEDA study: effectiveness of occupational therapy at home for older people with dementia and their caregivers-the design of a pragmatic randomised controlled trial evaluating a Dutch programme in seven German centres. BMC Geriatr 2009;9:44.

27. Teunisse S, Derix MM. The interview for deterioration in daily living activities in dementia: agreement between primary and secondary caregivers. Int Psychogeriatr 1997:9(Suppl 1):155-62.

28. Chapparo C, Ranka J. The PRPP System of Task Analysis: User's Training Manual. Research Edition. Sydney: OP Network, 2006.

29. Moniz-Cook E, Vernooii-Dassen M, Woods R, et al; INTERDEM group. A European consensus on outcome measures for psychosocial intervention research in dementia care. Aging Ment Health 2008:12:14-29.

30. Wolfson C, Moride Y, Perrault A, et al. Drug Treatments for Alzheimer's Disease. II. A Review of Outcome Measures in Clinical Trials. Ottawa: Canadian Coordinating Office for Health Technology Assessment (CCOHTA), 2000.

31. Faul $F$, Erdfelder $E$, Lang AG, et al. $G^{*}$ Power 3: A flexible statistical power analysis for the social, behavioral, and biomedical sciences. Behav Res Methods 2007;39:175-91. G*POWER free software. http://www.psycho.uni-duesseldorf.de/abteilungen/aap/gpower3/.

32. Allison P. Missing Data. Thousand Oaks, CA: Sage, 2002.

33. Plassman BL, Williams JW Jr, Burke JR, et al. Systematic review: factors associated with risk for and possible prevention of cognitive decline in later life. Ann Intern Med 2010;153:182-93.

34. Howell DC. Statistical Methods for Psychology. 4th edn. Belmont, CA Wadsworth, 1997.

35. Coley N, Gardette V, Cantet C, et al. How should we deal with missing data in clinical trials involving Alzheimer's disease patients? Curr Alzheimer Res 2011;4:421-33.

36. Zwarenstein M, Treweek S, Gagnier JJ, et al; CONSORT group Pragmatic Trials in Healthcare (Practihc) group. Improving the reporting of pragmatic trials: an extension of the CONSORT statement. BMJ 2008;337:a2390.

37. Gitlin LN, Winter L, Dennis MP, et al. A biobehavioral home-based intervention and the well-being of patients with dementia and their caregivers: the COPE randomized trial. JAMA 2010;304:983-91.

38. Smits $\mathrm{CH}$, de Lange J, Dröes RM, et al. Effects of combined intervention programmes for people with dementia living at home and their caregivers: a systematic review. Int J Geriatr Psychiatry 2007;22:1181-93.

39. Rieckmann N, Schwarzbach C, Nocon M, et al. Concepts of care for people with dementia. GMS Health Technol Assess 2009;5:Doc01. http://www.egms.de/static/en/journals/hta/2009-5/hta000063.shtml (accessed 29 Jul 2011) (in German).

40. Gauthier S, Lopez OL, Waldemar G, et al. Effects of donepezil on activities of daily living: integrated analysis of patient data from studies in mild, moderate and severe Alzheimer's disease. Int Psychogeriatr 2010;22:973-83. 